\title{
Shared Decision-Making and Outcomes in Type 2 Diabetes: A Systematic Review and Meta-Analysis
}

\section{Running Title: Shared decision-making and diabetes outcomes}

Michael Saheb Kashaf, MSc

School of Medicine, Johns Hopkins University School of Medicine, Baltimore, USA

Elizabeth Tyner McGill, MSc

Health Services Research and Policy, London School of Hygiene \& Tropical Medicine, London, UK

Zackary Dov Berger, MD, PhD

Division of General Internal Medicine, Johns Hopkins University School of Medicine, Baltimore, USA

Corresponding Author at

733 N Broadway, Baltimore, MD 21205 USA

Telephone: $240-640-4412$

Fax Number: 410-614-3386

E-mail: mskashaf@jhmi.edu

Number of Tables: 1

Number of Figures: 3

Highlights:

- SDM broadly found associated with improved process of care and unchanged clinical outcomes.

- Meta-analysis revealed an association between SDM and improved decision quality.

- Meta-analysis revealed a null association between SDM and glycemic control.

ABSTRACT

Objective. Type 2 diabetes is a chronic disease which necessitates the development of a therapeutic alliance between patient and provider. This review systematically examines the association between treatment shared decision-making (SDM) and outcomes in diabetes. Methods. A range of bibliographic databases and gray literature sources was searched. Included studies were subjected to dual data extraction and quality assessment. Outcomes were synthesized using meta-analyses where reporting was sufficiently homogenous or alternatively synthesized in narrative fashion. Results. The search retrieved 4,592 records, which were screened by title, abstract, and full text to identify 16 studies with a range of study designs and populations. We found evidence of an association between SDM and improved decision quality, patient knowledge and patient risk perception. We found little evidence of an association between SDM and glycemic control, patient satisfaction, quality of life, medication adherence or trust in physician. Conclusions. This work elucidates the potential clinical utility of SDM interventions in the management of Type 2 Diabetes and helps inform future research on the topic. Practice Implications. A more complete understanding of the associations between SDM and outcomes will guide and motivate efforts aimed at improving uptake of the SDM paradigm.

INTRODUCTION 
Clinical decision-making is beset by competing demands. Clinicians must uphold patient autonomy while acting in patients' best interest. There is a knowledge gap in both directions: While physicians usually have access to more clinical knowledge, patients know more about the ways decisions are likely to interact with their values, preferences and resources.

Shared decision-making (SDM) is an approach to clinical decision-making in which patients and practitioners jointly consider clinical factors and patient preferences to arrive at a decision based on mutual agreement $[1,2]$. The approach aims to bridge the information gap between patients and clinicians while upholding the ethical imperative toward patient autonomy. SDM was promoted by the influential Institute of Medicine (IOM) report, Crossing the Quality Chasm, [3] and has since gained wide acceptance as an indispensable component of patient-centered care [4]. Charles et al. offered the first and most influential framework for SDM. They defined the construct in relation to four criteria: 1. Involvement of at least two parties, 2. Mutual contribution to treatment deliberation, 3. Bilateral information exchange, 4. Mutual agreement [2]. In practice, however, most measures and interventions relating to SDM have focused on the second element of the Charles et al.defintion - mutual participation of the patient and clinician in the decision process.

SDM interventions have taken a number of approaches - including decision aids that present different options, videobased tools that address specific decisions or overall disease management and directly administered workshops that educate and empower patients to engage in decision-making [4-6]. These diverse approaches are united by a shared goal of increasing patient participation in the decision-making process.

SDM in Diabetes

Type 2 diabetes has a prevalence of $9.3 \%$ in the United States and is among the leading causes of mortality and morbidity [7]. In most cases, the disease requires lifelong management and simultaneous pharmacologic, behavioral and lifestyle interventions. Therefore, an understanding of the patient's values, preferences and life context is of great relevance [8]. The earliest antecedents of SDM - centered on the concept of mutual participation - recognized the unique synergies between the paradigm and the care of chronic diseases [9]. SDM interventions have been associated with increased patient knowledge, greater patient decision satisfaction, improved communication, less patient indecision, better congruence between patient values and decisions, improved patient risk perception, and improved decision quality in clinical care for a variety of conditions [10]. Moreover, SDM has been specifically linked with better diabetes self-management behaviors [11].

The evidence supporting approaches to diabetes treatment is sparse and of poor quality. One study has estimated that $40 \%$ of the diabetes guidelines espoused by the Endocrine Society are backed by low quality evidence [12]. Moreover, nearly $80 \%$ of patients with diabetes are estimated to suffer from at least one comorbid condition [13]. The evidence surrounding diabetes management is uncertain and treatment plans will need to account for individual patient comorbidities. These factors create opportunities for decision-making paradigms that attempt to capture patient factors and preferences. There is empirical evidence that patients differ from clinicians in their priorities regarding diabetes care [14]. This means that even robust guideline recommendations are complicated by individual patient needs, suggesting that personalized management approaches are necessary. The most recent guidelines from the American Diabetes Association (ADA) and the European Association for the Study of Diabetes (EASD) recognize this relevance and endorse a patient-centered approach to the condition [15] .

There have been several attempts to summarize the literature examining the impact of SDM in diabetes [16-18]. However, these studies limit SDM to decision aids and include only randomized trials. The broader shared decisionmaking literature has reached a consensus that SDM is not limited to decision aids alone; the construct can be operationalized using a variety of approaches and tools. These include video and instruction-based tools that build generic decisional self-efficacy, without addressing the specific decision scenarios addressed in decision aids [19, 20]. Further, we believe that limiting a review to RCTs fails to include much relevant information from observational studies. These studies can contribute to causality claims regarding SDM and diabetes outcomes. Evidence can be derived from many methodologies, and for a complicated area such as the application of SDM to diabetes care, studies of many designs are potentially relevant. 
This review aims to summarize and, where possible, quantitatively synthesize the evidence examining the association between SDM and outcomes in diabetes, examining both observational and experimental designs and summarizing evidence relevant to both process of care and clinical outcomes. Our goal is a comprehensive assessment of the impact of SDM in diabetes care to better understand the effects of this paradigm on process of care and clinical outcomes.

\section{METHODS}

\section{Data Sources and Searches}

In June and July 2016, 12 bibliographic databases and gray literature sources were searched using search terms for SDM and Type 2 Diabetes. The sources searched included the Cochrane Database of Systematic Reviews, Embase, MEDLINE, PsycINFO, CINAHL Plus, PsycEXTRA, Open Grey, New York Academy of Medicine Grey Literature Report, RAND Corporation, National Institute for Health and Care Excellence, Institute of Medicine, and Google. The searches were not limited by date or language. In addition, the journals The Diabetes Educator and Diabetes Care were hand searched from February 1990 to August 2016 and January 1990 to August 2016, respectively. Finally, all references from included studies were screened for additional relevant studies. The full search strategy is presented in the supplementary material. Studies assessing SDM in Type 2 Diabetes and quantitative outcomes were included in this review.

\section{Study Selection}

Inclusion criteria. All retrieved studies were evaluated according to title and abstract for adherence to the following pre-specified eligibility criteria. Those passing this initial screening process were subsequently screened with the full text.

Participants: Was the study population composed of adults (18 years) with a clinical diagnosis of type 2 diabetes?

Setting: Did the study concern decision-making within any context of diabetes treatment or management?

Explanatory variable: Did the study measure or manipulate patient participation in diabetes decision-making?

Outcomes: Did the study measure process of care or clinical outcomes relevant to diabetes management? The aim of the review was to examine how SDM impacts diabetes management. Measures that did not pertain to patient care, such as assessments of physician satisfaction, were not extracted. Clinical outcomes that did not directly pertain to diabetes were also excluded.

Study design: Did the study use a comparative and longitudinal design? Noncomparative designs such as case series and exploratory research were excluded from the review. Non-longitudinal studies such as cross-sectional surveys were excluded.

Ten percent of abstracts ( $n=461$ ) were independently screened by 2 reviewers; differences were resolved by discussion. The decision to dual screen $10 \%$ of reviewed abstracts was driven by resource limitations. The review protocol included the provision that the discovery of significant and systematic differences at this stage of the review would lead to a reassessment of the utilized eligibility criteria. The remaining references were screened by 1 reviewer. Any queries were discussed by both reviewers and resolved by consensus. All outstanding disagreements were resolved through the involvement of a third, independent reviewer.

\section{Data Extraction and Quality Assessment}

Data extraction used standardized data categories drawn from a Cochrane review [10]. The tool extracted data on each study's design, setting, participants, intervention/control, and outcomes. Dual, independent quality appraisal and data extraction were conducted to assess reliability and then all remaining studies were checked for accuracy. All outcome measures addressed in 3 or more studies were addressed in text and, where reporting was sufficiently homogenous, subjected to a meta-analysis. The remainder of the reported outcomes were summarized in tabular form. The review extracted outcomes within three categories - Clinical, Psychosocial and Behavioral. Clinical outcomes were direct or 
proxy measures of diabetes management and the effectiveness of disease control. Psychosocial outcomes captured patient perceptions of the quality of diabetes care, tested patient understanding of diabetes management and assessed measures of patient quality of life. Behavioral outcomes captured the extent and effectiveness of patient participation in diabetes management. All statistical analysis was conducted using Review Manager 5.3 software [21]. For outcome measures only addressed in tabular form, the results noted the direction of the observed effects in conjunction with the appraised methodological quality.

Meta-analysis was initially performed using a fixed effects model and a random effects model was adopted if $I^{2}>50 \%$. An overall effect was reported if the meta-analysis test for overall effect was significant at $(\alpha=0.05)$. Otherwise, the $p$-value for the test was reported. Outcome measures which could not be synthesized in meta-analyses were discussed in text using narrative synthesis focusing on assessed methodological quality. The potential for publication biases in studies included in the meta-analyses was investigated using funnel plots.

The design characteristics of all included studies were assessed using a quality assessment tool adapted from the validated, frequently used Downs and Black checklist [22] (supplementary material). The tool was selected for its applicability to studies of both experimental and observational design. The modified checklist scores study quality on a scale 0-27 and assesses the domains of study reporting, external validity, internal validity and statistical power. The modified checklist assesses the domains of study reporting, external validity, internal validity and statistical power and scores study quality on a scale of 0-27. Studies are categorized as "Low" quality (0-15), "Intermediate" quality (16-21) and "High" quality (22-27).

\section{RESULTS}

\section{Search Results}

Sixteen publications were included in the analysis. See Figure 1.

\section{Study Characteristics}

The reviewed literature included 14 experimental studies, including 13 randomized controlled trials (RCT) and 1 quasiexperimental study. The included quasi experimental study was a pilot feasibility study[23]. Two observational studies were also included, both were prospective cohort studies. The reviewed studies were conducted in the US $(n=11)$, Germany $(n=1)$, Greece $(n=1)$, the Netherlands $(n=1)$, Spain $(n=1)$ and the UK $(n=1)$. Process of care outcomes and clinical outcomes were abstracted from the included studies. Outcomes extracted from at least three studies included: patient knowledge ( $n=10$ studies), HbA1c $(n=9)$, decision quality $(n=8)$,$) , risk perception (n=6)$, satisfaction ( $n=6)$, quality of life $(n=5)$, medication adherence $(n=4)$, and trust $(n=3)$. The remainder of the outcome measures were addressed in two or fewer studies. Several SDM interventions were evaluated in multiple publications. The "Statin Choice" decision aid was evaluated in 5 publications, while the "Diabetes Medication Choice" decision aid was evaluated in 3 publications. The "Statin Choice" decision aid presents personalized patient information on cardiovascular risk factors and baseline 10-year cardiovascular risk in fostering active patient participation regarding statin use in diabetes management. The "Diabetes Medication Choice" decision aid describes 5 anti-hyperglycemic drugs, their treatment burden, and impact on HbA1c in facilitating SDM regarding diabetes treatment. The characteristics and findings of the included literature are summarized in Table 1.

\section{Quality Assessment}

The quality tiers used for categorization of studies as "Low", "Intermediate" and "High" quality are presented in the supplementary materials. The dual-reviewed quality assessments for all included studies are listed in Table 1. The included studies did not uniformly include validated checks of intervention fidelity or, for the observational studies, measurements of fidelity to the exposure. In the reviewed literature, a total of 6 studies did not include such checks $[19,29,30,31,33,34]$.

Investigation of Publication Biases 
The funnel plots for the $\mathrm{HbA} 1 \mathrm{c}$ and decision conflict scale meta-analyses are presented in the supplementary materials. The HbA1c plot contains too few points to robustly detect a publication bias but the asymmetry in the plot strengthens this possibility. The relative symmetry of the decision conflict scale funnel plot, on the other hand, weakens the possibility of a publication bias.

\section{Participant Characteristics}

A total of 2296 participants were involved in the included studies; 1759 participants (76.6\%) were included in studies with an experimental design, and 537 participants (23.4\%) were enrolled in studies with an observational design. Reporting of demographic information was variable. Reporting of age and gender distribution was mostly complete, while studies reported less frequently on variables such as ethnicity and baseline glycemic control. 
*: Scored on a scale from 0-27 using an adapted version of the Downs and Black checklist. $\mathrm{F}=$ Female.

†: The cited sample size is the number of participants could be reached at the final time point assessed in the study. All deviations from this convention are explicitly indicated.

‡: Only outcomes relevant to diabetes management were abstracted. "CO" refers to clinical outcomes; "BO" refers to behavioral outcomes, "PS" refers to psychosocial outcomes.

П: Outcome findings were reported using the following convention: "+": Improved outcomes, "0": No difference, "-": Worse outcomes.

$\otimes:$ "Decision type" refers to the decision category addressed by the study intervention. Some studies targeted specific decisions ("Specific"), while others more broadly addressed diabetes decisional efficacy ("Generic").

Ф: Weymiller et al. (2007) and Nannenga et al. (2009) report different outcomes measures for the same study population and sample.

$\Psi:$ Conference poster presentation 
Main Findings

\section{Clinical Outcomes}

Impact on Glycemic Control

Seven experimental and 2 observational studies assessed the association between SDM and glycemic control using the $\mathrm{HbA} 1 \mathrm{C}$ as the surrogate measure $[19,20,23,26,28,29,32,35,36]$. Among the 7 experimental studies, 1 noted a positive association and the remainder noted null associations. Among the 2 observational studies, 1 found a positive association and the other found no association.

Six studies with experimental designs and continuous measures of HbA1c were initially combined in a fixed effects meta-analysis (Figure 2). One experimental study could not be included in the meta-analysis due to its categorical outcome reporting. The finding of $\mathrm{I}^{2}>50 \%$ promoted the alternate use of a random effects model. The test for overall effect showed little evidence for an association between SDM interventions and $\mathrm{HbA1c}(\mathrm{P}=0.23)$.

Figure 2. Random effects meta-analysis of six experimental studies examining the SDM-HbA1C association.

\section{Psychosocial Outcomes}

Impact on Quality of Life (QOL)

Quality of life was reported in 1 observational and 4 experimental studies, with $\mathbf{3}$ using validated measures $[\mathbf{2 0}, \mathbf{3 0}, \mathbf{3 5}]$. The observational study performed a composite assessment of QOL, separately examining physical and mental functioning, and found a positive association for mental and a null relationship for physical functioning [35]. Among the experimental studies, one examined a composite measure of QOL and found a positive association for physical functioning and a null relationship for psychological functioning [20]. The three remaining experimental studies all assessed measures of general QOL; two found no relationship between SDM and QOL [26, 30], while one found an association between SDM and enhanced QOL [19].

Among these studies, two reported measures of self-perceived health status [26, 30], two reported assessments of health-related quality of life $[19,35]$ and only one met the definition of quality of life that includes assessment of general physical and psychological functioning [20].

\section{Impact on Patient Knowledge}

Patient knowledge was assessed by 10 included experimental studies. The studies used a heterogeneous set of measures of knowledge and assessed a variety of knowledge domains pertaining to diabetes. The majority of the utilized measures were ad hoc and unvalidated [20, $24,26-29,31,33,34]$; only one study reported using a validated measure of patient knowledge [19]. Among the 10 included studies, 6 noted a positive association between SDM and improved patient knowledge while 4 reported no association between the constructs. Some measures examined knowledge addressed on the decision aid while others made broader and more general assessments of patient diabetes knowledge. 
Impact on Decision Quality

Decision quality was assessed by 8 included experimental studies using the Decisional Conflict Sale (DCS) tool [37]. The DCS tool operationalizes decision quality using a validated questionnaire that assesses patient confidence regarding decision-making. The tool uses this decision confidence as measure of decisional self-efficacy and satisfaction. Among the 8 included studies, 3 found an association between SDM and improved decision quality while 5 others found no effect of SDM.

Seven studies with experimental designs and continuous measures of decision quality - all assessed using the DCS tool - were initially combined in a fixed effects meta-analysis. One study could not be included in the meta-analysis because measures of spread and the final analysed sample were not reported. The finding of $I^{2}>50 \%$ promoted the alternate use of a random effects model. This analysis is shown in Figure 3. The test for overall effect showed strong evidence for an association between SDM and better DCS outcomes [lower DCS scores] ( $p=$ $0.02)$.

Figure 3. Random effects meta-analysis of seven experimental studies examining the SDM-DCS association.

Impact on Risk Perception

Risk perception - referring to patients' awareness and understanding of their statistical risks under varying treatment scenarios [38] - was assessed in 6 experimental studies, all with unvalidated measures of the construct. Four of the studies assessed the construct by asking patients to estimate their risk of cardiovascular disease over 10 year with and without statin use $[24,27,29,34]$, the two remaining studies conducted disparate assessments of patient risk estimation [28, 32] . Two studies found evidence for a positive association between SDM and diabetes risk perception. Three studies had mixed findings where at least one but not all subscales of the risk perception measure showed a positive association. A single study showed a null association.

Impact on Patient Satisfaction

Five experimental studies and one observational study assessed patient satisfaction; three used validated measures $[19,20,30]$ and two used unvalidated measures of the construct $[31,35]$. Among the experimental studies, one found a positive association while 4 reported null effects. The sole observational study found a positive association between SDM and satisfaction. The studies examined a wide range of dimensions of satisfaction including overall satisfaction [20], visit satisfaction [35], satisfaction with decision-making [34], satisfaction with diabetes care [30], satisfaction with the interpersonal style of physician [19], and satisfaction with both communication and decision-making [31].

Impact on Trust of Physician

Trust in the physician was assessed in one observational and 2 experimental studies, all using validated measures $[25,26,35]$. The two experimental studies used the validated 9-item Trust in Physician scale while the included observational study used an 11-item validated measure of 
trust. The two experimental studies both failed to find an association between SDM and trust in the physician, while the sole observational study noted a positive association.

\section{Behavioral Outcomes}

Impact on Medication Adherence

Levels of adherence to prescribed medications were assessed in 5 experimental studies. Three used self-reported measures [24, 27, 34], one used pharmacy records [29] and one used both types of measures [26]. Three studies examined adherence to statins [24, 27, 34], One study examined adherence to antihyperglycemic medication [26], and one examined adherence to both statins and antihyperglycemic medication [29]. The three studies of statin adherence and the sole study of both statin and antihyperglycemic adherence all found null associations while the study examining antihyperglycemic medication adherence noted a relationship between SDM and improved adherence.

\section{DISCUSSION AND CONCLUSION}

Discussion

\section{Analysis \& Inferences}

Despite the weight of evidence and a growing consensus regarding its centrality in patientcentered care, SDM remains underutilized in diabetes care [39]. Barriers include time constraints and the perception of a lack of applicability of SDM due to situational or patient factors [40]; while two important facilitators include perceptions of a positive impact on care processes and clinical outcomes [40].

Our finding of improved process of care outcomes coupled with unchanged clinical outcomes raises two possible interpretations: either the finding of improved process of care outcomes is due to measurement bias or improvements in process of care outcomes did not translate to improved clinical measures, thus necessitating a re-examination of theoretical models linking SDM and improved outcomes. While our findings might reinforce the implementation of SDM in diabetes care, methodological issues and multiple sources of bias caution against a simple interpretation of the relationship between processes of care and clinical outcomes.

This literature is rich in potential methodological sources of bias. Among included experimental studies, $33 \%$ (21/65) of study outcome showed positive findings while $71 \%(5 / 7)$ of observational study outcomes showed positive findings. This discrepancy likely reflects the lower appraised methodological quality of the observational literature and the greater likelihood of bias in these designs. We examined time cohort effects by stratifying study outcomes into those reported before or after the year 2010. The selected year was chosen in order to dichotomize the retrieved studies into two time cohorts each containing a roughly equal number of studies. The approach examined the fraction of positive outcome findings in each stratum and found little evidence of an effect.

Further, experimental studies largely failed to pair their interventions with control conditions where patients received adequate time and attention. In this context, subjective process of care outcome measures such as decision quality were more vulnerable to measurement bias. 
There were also differences in assessment, with process of care measures generally assessed immediately after the initial encounter and clinical outcomes assessed after a follow-up period. It is therefore unclear whether the differences in findings can be partially attributed to time effects and limitations in the durability of the intervention effects. Among observational studies, patient reports of SDM use are likely correlated with a range of patient-centred care metrics. Moreover, reports of participation in decision-making may partly reflect patient resources and capacities. Patients engaged in the decision-making process may be more educated and healthy enough to participate.

\section{Strengths and Limitations}

A sensitive search strategy and inclusive selection criteria improve the validity of the derived findings. The review also benefited from use of a validated quality assessment tool and external standard drawn from the PRISMA statement [41]. The broad inclusion of both process of care and clinical outcomes also allows for a comprehensive assessment of the impact of SDM on diabetes outcomes.

There are several features that distinguish this work from previous efforts. First, the review includes both observational and experimental designs. Second, the review includes all SDM intervention approaches and goes beyond a consideration of only decision aids or any single means of implementing SDM. Third, the study performs meta-analyses where outcome reporting is sufficiently homogenous and thereby allows for a more systematic and reproducible means of synthesizing literature findings. Lastly, the work addresses a broad range of SDM outcomes - spanning both process of care and clinical outcome. This range enables more comprehensive assessment of the impact of SDM on diabetes care.

A number of limitations also merit consideration. First, the variability in the operationalization of SDM limits the generalizability of the meta-analyses [42]. Second, the heterogeneity and scope of the SDM construct limits search sensitivity. Third, as seen in Table 1, many included studies either used unvalidated measures of decision participation or failed to report any such measures. This means that included studies did not uniformly include a check of intervention fidelity. This limits the interpretability of the literature and limits the ability to assess the true impact on shared decision making on diabetes outcomes. Finally, existing research suggests that diversity is a potentially important variable moderating the impact of SDM on diabetes outcomes [43]. The extant literature, however, fails to provide stratified datasets that can elucidate this association. This represents a limitation of both the source literature and this review.

\section{Conclusion}

The review examined the association between SDM and process of care and clinical outcomes in Type 2 diabetes. We found substantial evidence of an association between SDM and improved decision quality, patient knowledge and patient risk perception. We found little evidence of an association between SDM and glycemic control, patient satisfaction, quality of life, medication adherence or trust in physician.

Practice Implications 
The findings of this review suggest SDM is associated with improvements in several relevant diabetes outcome metrics. SDM remains underutilized in clinical practice and this review should therefore add impetus to efforts to encourage greater uptake of the paradigm[44]. Future work will also need to undertake implementation studies that examine practical approaches for bringing SDM to the clinic.

\section{Author Contributions}

M.S.K. developed search strategy, implemented search, conducted study selection, performed data extraction \& quality assessment, performed data analysis and wrote manuscript.

E.T.M developed search strategy, conducted study selection, performed data extraction \& quality assessment, reviewed/edited manuscript.

Z.B. developed search strategy, reviewed/edited manuscript.

\section{Acknowledgements}

We thank Jaime Blanck of the Welch Medical Library at Johns Hopkins University, for assistance with the development of the search strategy.

The authors declare that there are no conflicts of interest.

Guarantor of submission: The corresponding author is the guarantor of submission.

Funding: This review did not receive any external funding.

Figure Legends 


\section{PRISMA 2009 Flow Diagram}

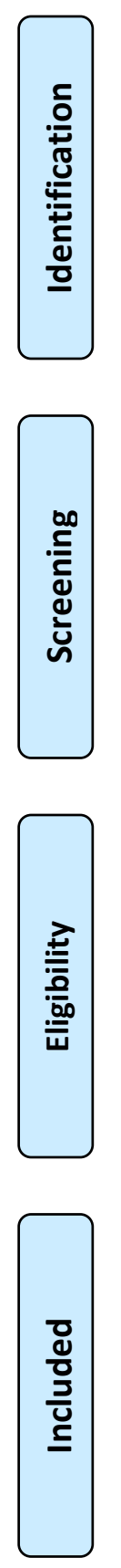

Records identified through database searching (CINAHL, Cochrane, EMBASE, MedLine, PsycINFO) $(n=3233)$
Additional records identified through other sources (gray literature, hand search, reference search) $(n=1359)$

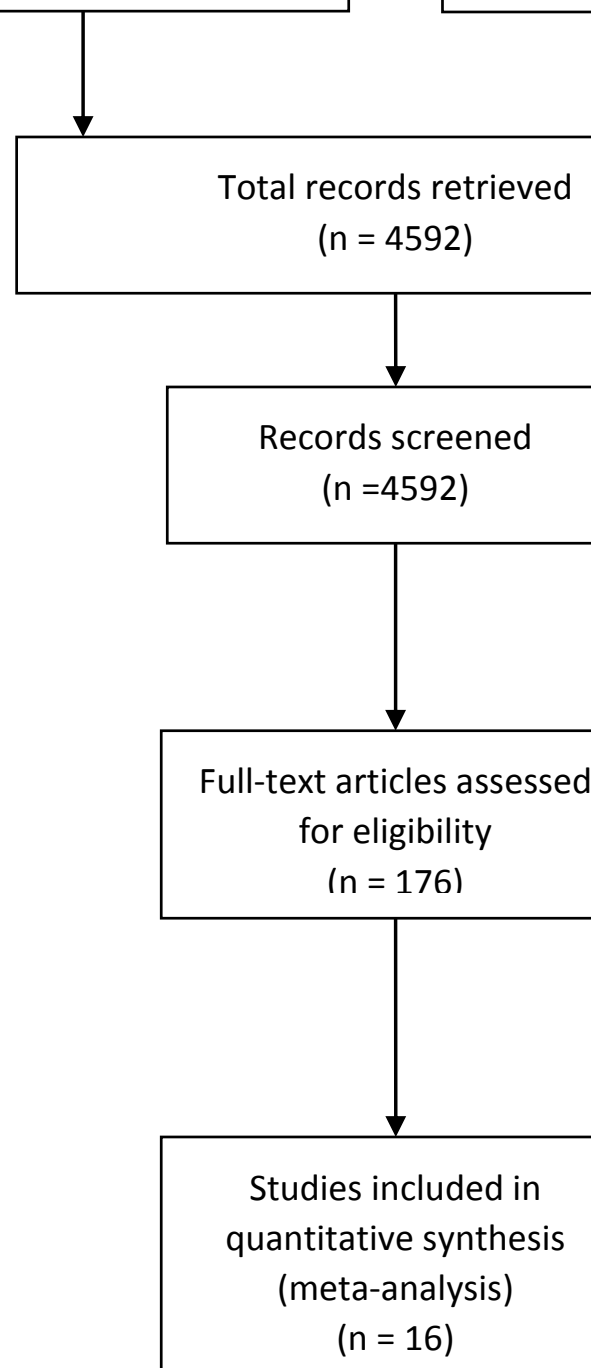

Records excluded as not meeting eligibility criteria following assessment by

Full-text articles excluded, with reasons $(n=160)$

Disease criterion $=4$

Intervention/exposure $=30$

No primary data $=43$

Duplicate $=55$

Figure 1. Flow of literature through the review. 


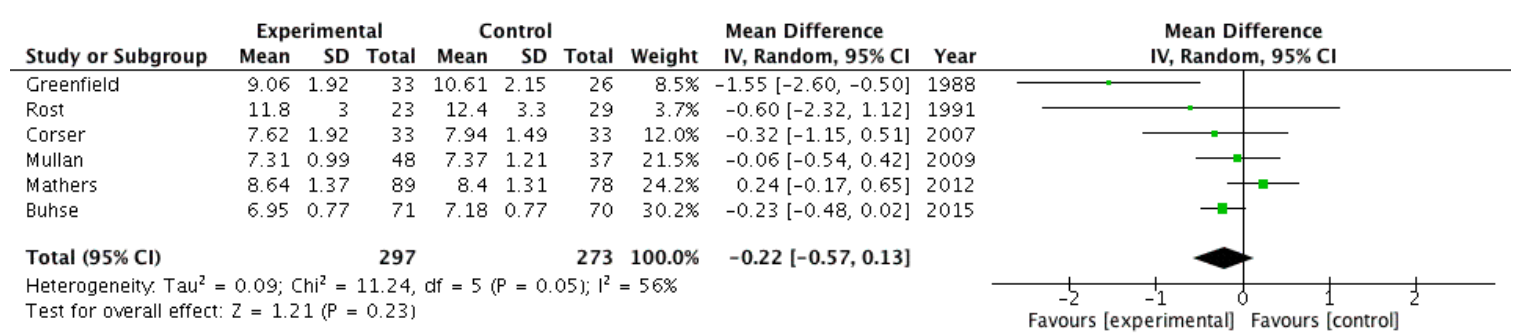

Figure 2. Random effects meta-analysis of six experimental studies examining the SDM-HbA1c association.

\begin{tabular}{|c|c|c|c|c|c|c|c|c|c|c|c|}
\hline \multirow[b]{2}{*}{ Study or Subgroup } & \multicolumn{3}{|c|}{ Experimental } & \multicolumn{3}{|c|}{ Control } & \multicolumn{3}{|c|}{ Mean Difference } & \multirow{2}{*}{\multicolumn{2}{|c|}{$\begin{array}{c}\text { Mean Difference } \\
\text { IV, Random, } 95 \% \mathrm{CI}\end{array}$}} \\
\hline & Mean & SD & Total & Mean & SD & Total & Weight & IV, Random, $95 \% \mathrm{CI}$ & Year & & \\
\hline Weymiller & -10.6 & 11.9 & 51 & 0 & 11.9 & 46 & $16.6 \%$ & $-10.60[-15.34,-5.86]$ & 2007 & & \\
\hline Mullan & 14.1 & 17.89 & 48 & 14.95 & 12.68 & 37 & $14.6 \%$ & $-0.85[-7.35,5.65]$ & 2009 & & \\
\hline Mathers & 17.4 & 12.6 & 89 & 25.2 & 14.9 & 78 & $17.2 \%$ & $-7.80[-12.02,-3.58]$ & 2012 & $\longrightarrow$ & \\
\hline Branda & 82.9 & 15.2288 & 53 & 81.2 & 21.2854 & 50 & $13.8 \%$ & $1.70[-5.48,8.88]$ & 2013 & & \\
\hline Karagiannis & 17.9 & 63.5808 & 101 & 25 & 75.34 & 103 & $4.9 \%$ & $-7.10[-26.22,12.02]$ & 2014 & & \\
\hline Bailey & -22.2 & 20.6 & 114 & -7.5 & 16.6 & 111 & $16.5 \%$ & $-14.70[-19.58,-9.82]$ & 2016 & $\longrightarrow$ & \\
\hline Perestelo-Perez & 23.89 & 16.75 & 78 & 23.77 & 14.83 & 77 & $16.4 \%$ & $0.12[-4.86,5.10]$ & 2016 & & \\
\hline Total $(95 \% \mathrm{CI})$ & & & 534 & & & 502 & $100.0 \%$ & $-5.74[-10.63,-0.85]$ & & & \\
\hline $\begin{array}{l}\text { Heterogeneity. Tau }{ }^{2} \\
\text { Test for overall effect }\end{array}$ & $\begin{array}{l}31.60 \\
z=2.3\end{array}$ & $\begin{array}{l}C h i^{2}=28 . \\
0(P=0.02\end{array}$ & $\begin{array}{l}74, d f= \\
2)\end{array}$ & $6(t$ & 00001 & $=79$ & & & & $\begin{array}{cc}-20 & -10 \\
\text { Favours [experiment }\end{array}$ & ]$^{0} \stackrel{10}{10}{ }^{1}{ }^{2}$ \\
\hline
\end{tabular}

Figure 3. Random effects meta-analysis of seven experimental studies examining the SDM-DCS association 


\section{References:}

1. Frosch, D.L. and R.M. Kaplan, Shared decision making in clinical medicine: past research and future directions. American journal of preventive medicine, 1999. 17(4): p. 285-294.

2. Charles, C., A. Gafni, and T. Whelan, Shared decision-making in the medical encounter: what does it mean?(or it takes at least two to tango). Social science \& medicine, 1997. 44(5): p. 681-692.

3. Plsek, P., Institute of Medicine. Crossing the Quality Chasm: A New Health System for the 21st Century. 2001, Washington, DC: National Academy Press.

4. Barry, M.J. and S. Edgman-Levitan, Shared decision making - the pinnacle of patient-centered care. New England Journal of Medicine, 2012. 366(9): p. 780-781.

5. Frosch, D.L., R.M. Kaplan, and V. Felitti, The evaluation of two methods to facilitate shared decision making for men considering the Prostate-specific antigen test. Journal of general internal medicine, 2001. 16(6): p. 391-398.

6. O'Connor, A.M., H.A. Llewellyn-Thomas, and A.B. Flood, Modifying unwarranted variations in health care: shared decision making using patient decision aids. Health Affairs, 2004: p. VAR63.

7. Control, C.f.D. and Prevention, National diabetes statistics report: estimates of diabetes and its burden in the United States, 2014. Atlanta, GA: US Department of Health and Human Services, 2014. 2014.

8. Montori, V.M., A. Gafni, and C. Charles, A shared treatment decision-making approach between patients with chronic conditions and their clinicians: the case of diabetes. Health Expectations, 2006. 9(1): p. 2536.

9. Brody, D.S., The patient's role in clinical decision-making. Annals of Internal Medicine, 1980. 93(5): p. 718-722.

10. Stacey, D., et al., Decision aids for people facing health treatment or screening decisions. The Cochrane Library, 2014.

11. Thorpe, C.T., et al., Facilitating healthy coping in patients with diabetes a systematic review. The Diabetes Educator, 2012: p. 0145721712464400.

12. Ospina, N.S., et al., Is the endocrine research pipeline broken? A systematic evaluation of the Endocrine Society clinical practice guidelines and trial registration. BMC medicine, 2015. 13(1): p. 187.

13. Lin, P.-J., et al., Multiple chronic conditions in type 2 diabetes mellitus: prevalence and consequences. The American journal of managed care, 2015. 21(1): p. e23-34.

14. Hares, T., et al., Diabetes care: who are the experts? Quality in Health Care, 1992. 1(4): p. 219-224.

15. Inzucchi, S.E., et al., Management of hyperglycemia in type 2 diabetes: a patient-centered approach position statement of the American Diabetes Association (ADA) and the European Association for the Study of Diabetes (EASD). Diabetes care, 2012. 35(6): p. 1364-1379.

16. Wilkinson, M.J., A.G. Nathan, and E.S. Huang, Personalized decision support in type 2 diabetes mellitus: current evidence and future directions. Current diabetes reports, 2013. 13(2): p. 205-212.

17. Serrano, V., et al., Shared decision-making in the care of individuals with diabetes. Diabetic Medicine, 2016. 33(6): p. 742-751.

18. Rodriguez-Gutierrez, R., et al., Shared decision making in endocrinology: present and future directions. The Lancet Diabetes \& Endocrinology, 2016.

19. Greenfield, S., et al., Patients' participation in medical care. Journal of general internal medicine, 1988. 3(5): p. 448-457.

20. Rost, K.M., et al., Change in metabolic control and functional status after hospitalization: impact of patient activation intervention in diabetic patients. Diabetes Care, 1991. 14(10): p. 881-889.

21. Collaboration, N.C.C.T.C., Review Manager (RevMan)[Computer program] Version 53. Copenhagen: The Nordic Cochrane Centre, The Cochrane Collaboration, 2014.

22. Downs, S.H. and N. Black, The feasibility of creating a checklist for the assessment of the methodological quality both of randomised and non-randomised studies of health care interventions. Journal of epidemiology and community health, 1998. 52(6): p. 377-384.

23. Corser, W., et al., A shared decision-making primary care intervention for type 2 diabetes. The Diabetes Educator, 2007. 33(4): p. 700-708.

24. Weymiller, A.J., et al., Helping patients with type 2 diabetes mellitus make treatment decisions: statin choice randomized trial. Archives of internal medicine, 2007. 167(10): p. 1076-1082.

25. Nannenga, M.R., et al., A treatment decision aid may increase patient trust in the diabetes specialist. The Statin Choice randomized trial. Health Expectations, 2009. 12(1): p. 38-44. 
26. Mullan, R.J., et al., The diabetes mellitus medication choice decision aid: a randomized trial. Archives of internal medicine, 2009. 169(17): p. 1560-1568.

27. Mann, D.M., et al., The Statin Choice decision aid in primary care: a randomized trial. Patient education and counseling, 2010. 80(1): p. 138-140.

28. Mathers, N., et al., Clinical effectiveness of a patient decision aid to improve decision quality and glycaemic control in people with diabetes making treatment choices: a cluster randomised controlled trial (PANDAs) in general practice. BMJ open, 2012. 2(6): p. e001469.

29. Branda, M.E., et al., Shared decision making for patients with type 2 diabetes: a randomized trial in primary care. BMC health services research, 2013. 13(1): p. 1.

30. Denig, P., et al., Effects of a patient oriented decision aid for prioritising treatment goals in diabetes: pragmatic randomised controlled trial. 2014.

31. Karagiannis, T., et al. Shared decision making in type 2 diabetes using the Diabetes Medication choice Decision Aid: preliminary results from a cluster-randomised trial. in DIABETOLOGIA. 2014. SPRINGER 233 SPRING ST, NEW YORK, NY 10013 USA.

32. Buhse, S., et al., An informed shared decision making programme on the prevention of myocardial infarction for patients with type 2 diabetes in primary care: protocol of a cluster randomised, controlled trial. BMC family practice, 2015. 16(1): p. 1.

33. Bailey, R.A., et al., Effect of a patient decision aid (PDA) for type 2 diabetes on knowledge, decisional selfefficacy, and decisional conflict. BMC health services research, 2016. 16(1): p. 1.

34. Perestelo-Pérez, L., et al., Effect of the statin choice encounter decision aid in Spanish patients with type 2 diabetes: A randomized trial. Patient education and counseling, 2016. 99(2): p. 295-299.

35. Lee, Y.-Y. and J.L. Lin, Do patient autonomy preferences matter? Linking patient-centered care to patient-physician relationships and health outcomes. Social science \& medicine, 2010. 71(10): p. 18111818.

36. Parchman, M.L., J.E. Zeber, and R.F. Palmer, Participatory decision making, patient activation, medication adherence, and intermediate clinical outcomes in type 2 diabetes: a STARNet study. The Annals of Family Medicine, 2010. 8(5): p. 410-417.

37. O'Connor, A.M., Validation of a decisional conflict scale. Medical decision making, 1995. 15(1): p. 25-30.

38. Slovic, P., Perception of risk: Reflections on the psychometric paradigm. 1992.

39. Stiggelbout, A.M., et al., Shared decision making: really putting patients at the centre of healthcare. Bmj, 2012. 344(S 28).

40. Légaré, F., et al., Barriers and facilitators to implementing shared decision-making in clinical practice: update of a systematic review of health professionals' perceptions. Patient education and counseling, 2008. 73(3): p. 526-535.

41. Moher, D., et al., Preferred reporting items for systematic reviews and meta-analyses: the PRISMA statement. Annals of internal medicine, 2009. 151(4): p. 264-269.

42. Makoul, G. and M.L. Clayman, An integrative model of shared decision making in medical encounters. Patient education and counseling, 2006. 60(3): p. 301-312.

43. Peek, M.E., et al., Race and shared decision-making: perspectives of African-Americans with diabetes. Social science \& medicine, 2010. 71(1): p. 1-9.

44. Holmes-Rovner, M., J. Gruman, and D.R. Rovner, Shared decision-making in the US-research \& development outpaces delivery. Zeitschrift für ärztliche Fortbildung und Qualität im GesundheitswesenGerman Journal for Quality in Health Care, 2007. 101(4): p. 254-258. 